\title{
EFFECT OF COMPOST, SALICYLIC AND ASCORBIC ACIDS TREATMENTS ON VEGETATIVE GROWTH AND FLOWERING OF GLADIOLUS GRANDIFLORUS CV. WHITE PROSPERITY
}

\author{
M.A.H. Abdou; A.A. El-Sayed; F.A. Attia and A.R.M. Khalil \\ Horticulture Dept., Fac. Agric., Minia Univ., Egypt.

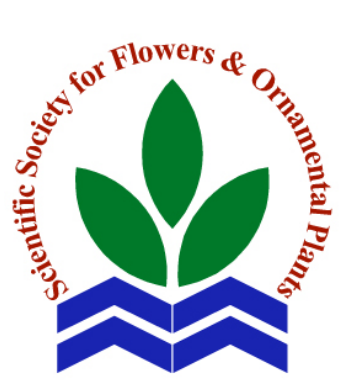 \\ Scientific J. Flowers \& \\ Ornamental Plants, \\ 1(3):223-231 (2014). \\ Received: \\ $4 / 11 / 2014$ \\ Revised by: \\ Prof. Dr. A.Z. Sarhan, \\ Cairo Univ. \\ ABSTRACT: A field experiment was carried out during the two \\ successive seasons of 2012/2013 and 2013/2014. The aim of this study \\ was to investigate the effect of compost at four levels $(0,7.5,10.0$ and \\ 12.5 ton/fed) in combination with salicylic and/or ascorbic acids, each \\ at 100 and $200 \mathrm{ppm}$, in addition to the control on growth and flowering \\ of Gladiolus grandiflorus cv. White Prosperity. \\ Results showed that leaf length, leaf area, number of leaves/plant, \\ leaves dry weight/plant, length of spike, rachis length, spike diameter, \\ number of florets/spike, lower floret diameter and single floret fresh \\ weight were gradually increased by increasing the levels of compost \\ with significant differences were detected between successive \\ treatments. Also, all salicylic and/or ascorbic acid treatments \\ significantly increased all vegetative growth and flowering parameters \\ in comparison with the control. Salicylic plus ascorbic one at $100 \mathrm{ppm}$ \\ for each, followed by ascorbic acid alone at $200 \mathrm{ppm}$ were more \\ effective in this concern. It was found also that the use of compost \\ (12.5 ton/fed) in combination with salicylic acid + ascorbic acid, each \\ at $100 \mathrm{ppm}$ or with ascorbic acid at $200 \mathrm{ppm}$ noticeably improved the \\ different vegetative growth characters and flowering parameters of \\ gladiolus plants.
}

Prof. Dr. S.M. Shahin, Hort. Res. Inst., ARC.

Key words: Gladiolus grandiflorus, cv. White Prosperity, compost, salicylic acid, ascorbic acid, vegetative growth, flowering.

\section{INTRODUCTION}

Gladiolus is derived from the native plants of South and Central Africa, as well as, the Mediterranean region. Gladiolus (Family Iridaceae) is a valuable and economic flowering bulb plant used as a landscape plant in the home gardens and in decoration as a lovely and rich colored cut flower spike with relatively long vase life (Hogan, 1990). In this study corms of Gladiolus gradiflorus cv. White Prosperity were chosen for its adaptability to the Egyptian environmental conditions, besides the possibility of exporting of its flowers could be increased.
Organic, salicylic and ascorbic acids are among the important agricultural treatments which have been proved to improve the vegetative growth and flowering aspects of gladiolus plants.

Many investigators revealed the importance of organic fertilization on the growth and flowering of gladiolus such as, Conte et al. (2001), Zaghloul and Moghazy (2001), Khan et al. (2002), Atta-Alla et al. (2003), Dongardive et al. (2007), Hassanein and El-Sayed (2009), Leonardo and Barbara (2011), Chandar et al. (2012) and Abdou et al. (2013).

The role of salicylic acid in improving vegetative growth and flowering parameters 
was revealed by Sajjad et al. (2014) on gladiolus, Khandaker et al. (2011) on red amaranths, Hajizadeh and Aliloo (2013) on Lilium longiflorum and Anwar et al. (2014) on tuberose.

The role of ascorbic acid in improving vegetative growth and flowering characters was also mentioned by Abdel Aziz et al. (2009), Abo Leila and Eid (2011) on gladiolus and Kasim and Adil (2014) on Freesia hybrida.

The aim of this work was to study the effect of compost, salicylic and ascorbic acids treatments on the vegetative growth and flowering of gladiolus cultivar, White Prosperity.

\section{MATERIALS AND METHODS}

A field experiment was carried out during the two successive seasons of 2012/2013 and 2013/2014 at the Nursery of Ornamental Plants, Faculty of Agriculture, Minia University to figure out the response of the Gladiolus grandiflorus cv. White Prosperity plants to plant compost, salicylic acid and ascorbic acid treatments.

The corms of gladiolus were obtained from Holland by Basiony nurseries, Cairo, Egypt. Average corm diameter was 2.8 and $3.1 \mathrm{~cm}$ and corm weight was 9.6 and $10.1 \mathrm{~g}$ for the first and second seasons, respectively. Corms were planted on October $1^{\text {st }}$ for both seasons in $1.5 \times 2.2 \mathrm{~m}$ plots containing 3 ridges, $50 \mathrm{~cm}$ apart. Corms were planted in hills, $20 \mathrm{~cm}$ apart (10 corms/ridge). Physical and chemical properties of the soil used are listed in Table (a). The split plot design with three replicates was followed in this experiment.

The four levels of compost fertilization treatments were considered as main plots and the seven treatments of salicylic and/or ascorbic acids the sub plots. The four levels of compost treatments were $0.0,7.5,10.0$ and $12.5 \mathrm{ton} / \mathrm{fed}$. The compost was added before planting during the soil preparation. Compost analysis is shown in Table (b).

The sub plots were as follows: control, salicylic acid at 100 or $200 \mathrm{ppm}$, ascorbic acid at 100 or $200 \mathrm{ppm}$, salicylic acid + ascorbic acid (each at 100 or $200 \mathrm{ppm}$ ). Salicylic and ascorbic acids were sprayed three times, one month and two months after planting and after flower cut.

\section{The following data were recorded:}

1- Vegetative growth characters just before flowering: leaf length $(\mathrm{cm})$, leaf area $\left(\mathrm{cm}^{2}\right)$, number of leaves/plant and dry weight of leaves/plant (g).

2- Flowering characters: length of spike $(\mathrm{cm})$, rachis length $(\mathrm{cm})$, spike diameter $(\mathrm{mm})$, number of florets/spike, lower floret diameter $(\mathrm{cm})$ and single floret fresh weight $(\mathrm{g})$.

The data of the two experiments were subjected to the statistical analysis of variance using MSTAT-C (1986). L.S.D. test at $5 \%$ was used to compared the average means of treatments.

Table a. Physical and chemical properties of the used soil in both seasons.

\begin{tabular}{|c|c|c|c|c|c|}
\hline \multirow{2}{*}{ Soil Character } & \multicolumn{2}{|c|}{ Value } & \multirow{2}{*}{ Soil Character } & \multicolumn{2}{|c|}{ Value } \\
\hline & $2012 / 2013$ & $2013 / 2014$ & & $2012 / 2013$ & 2013/2014 \\
\hline Sand \% & 28.98 & 28.90 & Available P \% & 15.67 & 15.58 \\
\hline Silt $\%$ & 29.87 & 30.64 & Exch. $\mathrm{K}^{+} \mathrm{mg} / 100 \mathrm{~g}$ & 2.85 & 2.64 \\
\hline Clay \% & 41.15 & 40.46 & Exch. $\mathrm{Ca}^{++} \mathrm{mg} / 100 \mathrm{~g}$ & 31.12 & 31.43 \\
\hline Soil type & Clay loam & Clay loam & Exch. $\mathrm{Na}^{+} \mathrm{mg} / 100 \mathrm{~g}$ & 2.51 & 2.50 \\
\hline Organic matter \% & 1.54 & 1.59 & $\mathrm{Fe}$ & 8.23 & 8.11 \\
\hline $\mathrm{Ca} \mathrm{CO}_{3} \%$ & 2.11 & 2.10 & $\mathbf{C u}$ & 2.01 & 2.00 \\
\hline pH (1: 2.5) & 7.75 & 7.71 & DTPA & 2.87 & 2.89 \\
\hline E. C. (m mhos/cm) & 1.08 & 1.06 & Ext. ppm & 8.11 & 8.15 \\
\hline Total N \% & 0.08 & 0.06 & & & \\
\hline
\end{tabular}


Table b. Chemical analysis of the compost used in the two seasons.

\begin{tabular}{lclc}
\hline Properties & Value & Properties & Value \\
\hline Organic carbon & 25.1 & Total P (\%) & 0.5 \\
Humidity (\%) & 25 & Total K (\%) & 1.0 \\
Organic matter (\%) & 44 & Fe (ppm) & 1750 \\
C/N ratio & 17.5 & Zn (ppm) & 60 \\
pH (1:2.5) & 8.0 & Mn (ppm) & 125 \\
E.C. (m. mhos/cm) & 5 & Cu (ppm) & 200 \\
Total N (\%) & 1.5 & & \\
\hline
\end{tabular}

\section{RESULTS AND DISCUSSION}

\section{1- Vegetative growth characters:}

Data presented in Tables (1 and 2) showed that leaf length and leaf area, number of leaves/plant and leaves dry weight/plant of gladiolus were significantly increased, in both seasons, due to the use of $7.5,10.0$ and 12.5 ton/fed compost in comparison with those of control plants. Moreover, the increase was gradually by the gradual increase in compost level. The increase in vegetative growth traits due to compost at levels of $7.5,10.0$ and 12.5 ton/fed over the control reached 5.67, 11.27 and $16.17 \%$ for leaf length, $11.75,56.86$ and $73.16 \%$ for leaf area, 13.05, 14.78 and $17.44 \%$ for leaf number and $12.89,14.90$ and $17.48 \%$ for leaves dry weight, respectively, in the first season and by 5.55 , 10.99 and $16.29 \%$ for leaf length, 33.38, 55.55 and $71.67 \%$ for leaf area, $11.73,14.64$ and $15.99 \%$ for number of leaves and 11.84 , 14.61 and 17.13 for leaves dry weight, respectively in the second one. Similar results were found by Conte et al. (2001), Khan et al. (2002), Chandar et al. (2012) and Abdou et al. (2013) on gladiolus.

The increase of vegetative growth resulting from using compost as organic fertilization might be due to that organic matter is considered an important factor for improving the physical, chemical and biological properties of the soil and consequently, increasing plant growth (Maynard, 1991).

Data in Tables (1 and 2) indicated that, leaf length, leaf area, number of leaves/plant and leaves dry weight of gladiolus were significantly increased, in both seasons, due to the use of salicylic and ascorbic acids, each at 100 or $200 \mathrm{ppm}$ either used separately or together in comparison with control. The combined treatment of salicylic acid (100 ppm) plus ascorbic acid (100 ppm) followed by treatment of ascorbic acid (200 ppm) seemed to be more effective than other treatments. In conformity with these results were those detected by Sajjad et al. (2014) on gladiolus and Anwar et al. (2014) on tuberose regarding the effect of salicylic acid. Moreover, Abdel Aziz et al. (2009) and Abo Leila and Eid (2011) on gladiolus concerning the influence of ascorbic acid.

Ascorbic acid is the most abundant antioxidant which protects plant cells, or antioxidant defense, photoprotection and regulation of photosynthesis and growth (Blokhina et al., 2003). The ameliorative effect of salicylic acid on growth of plants under a biotic stress conditions may have been due to role in nutrient uptake, water relations, stomatal regulation, photosynthesis and growth (Arfan et al., 2007).

The interaction between compost, salicylic and/or ascorbic acids treatments was significant in the two seasons for all previous characters. The maximum values were obtained due to fertilizing the soil of gladiolus with high level of compost (12.5 ton/fed) and spraying plants with salicylic acid $(100 \mathrm{ppm})+$ ascorbic acid $(100 \mathrm{ppm})$ or ascorbic acid at $200 \mathrm{ppm}$.

\section{2- Flowering parameters:}

Data presented in Tables (3 and 4) indicated that all compost levels caused a significant increase in length of spike, rachis length, spike diameter, number of florets/spike, lower floret diameter and single floret fresh weight, in the two seasons, in comparison with those of untreated plants. The means of flowering parameters were gradually increased according to the increase in levels of compost fertilizer. The application of compost at high level (12.5 ton/fed) resulted the highest values of various flowering traits. These results are in close with those obtained by Zaghloul and Moghazy (2001), Pimpini and Zanin (2002), 
Table 1. Effect of compost, salicylic and ascorbic acid treatments on leaf length (cm), leaf area $\left(\mathrm{cm}^{2}\right)$ and number of leaves/plant of Gladiolus grandiflorus cv. White Prosperity plants during 2012/2013 and 2013/2014 seasons.

\begin{tabular}{|c|c|c|c|c|c|c|c|c|c|c|}
\hline \multirow{3}{*}{$\begin{array}{c}\text { Salicylic and } \\
\text { ascorbic acids } \\
\text { treatments (B) } \\
\end{array}$} & \multicolumn{10}{|c|}{ Compost levels (ton/feddan) (A) } \\
\hline & \multicolumn{5}{|c|}{$1^{\text {st }}$ Season } & \multicolumn{5}{|c|}{$2^{\text {nd }}$ Season } \\
\hline & $\mathbf{0}$ & 7.5 & 10.0 & 12.5 & Mean (B) & $\mathbf{0}$ & 7.5 & 10.0 & 12.5 & Mean (B) \\
\hline & \multicolumn{10}{|c|}{ Leaf length $(\mathrm{cm})$} \\
\hline Control & 53.21 & 56.45 & 59.35 & 61.96 & 57.74 & 54.32 & 57.56 & 60.46 & 63.07 & 58.85 \\
\hline Sal. at 100 ppm & 55.02 & 58.36 & 61.37 & 64.08 & 59.71 & 56.32 & 59.57 & 62.58 & 65.28 & 60.94 \\
\hline Sal. at 200 ppm & 56.93 & 60.27 & 63.28 & 65.99 & 61.62 & 58.14 & 61.48 & 64.38 & 67.08 & 62.77 \\
\hline Asc. at 100 ppm & 55.14 & 58.48 & 61.64 & 64.99 & 60.02 & 56.34 & 59.78 & 62.95 & 66.28 & 61.34 \\
\hline Asc. at 200 ppm & 57.19 & 60.51 & 63.63 & 66.25 & 61.89 & 58.48 & 61.80 & 64.92 & 67.54 & 63.19 \\
\hline (Sal.+Asc.) at $100 \mathrm{ppm}$ & 59.38 & 62.82 & 65.83 & 68.44 & 64.12 & 60.69 & 64.13 & 67.14 & 69.75 & 65.43 \\
\hline (Sal.+Asc.) at $200 \mathrm{ppm}$ & 57.11 & 59.43 & 63.26 & 66.00 & 61.45 & 58.41 & 60.71 & 64.55 & 67.30 & 62.74 \\
\hline Mean (A) & 56.28 & 59.47 & 62.62 & 65.38 & & 57.53 & 60.72 & 63.85 & 66.90 & \\
\hline \multirow[t]{2}{*}{ L.S.D. at 5\% } & \multicolumn{2}{|c|}{ A: 2.55} & B: 1.10 & \multicolumn{2}{|c|}{ AB: 2.20} & \multicolumn{2}{|c|}{ A: 2.81} & B: 1.12 & \multicolumn{2}{|c|}{ AB: 2.24} \\
\hline & \multicolumn{10}{|c|}{ Leaf area $\left(\mathrm{cm}^{2}\right)$} \\
\hline Control & 46.2 & 60.6 & 70.6 & 77.5 & 63.73 & 46.7 & 61.3 & 71.3 & 78.3 & 66.65 \\
\hline Sal. at 100 ppm & 49.6 & 67.4 & 78.5 & 86.4 & 70.48 & 50.1 & 68.1 & 79.3 & 87.3 & 71.20 \\
\hline Sal. at 200 ppm & 55.5 & 75.1 & 87.6 & 96.7 & 78.73 & 56.1 & 75.1 & 88.5 & 97.7 & 79.35 \\
\hline Asc. at 100 ppm & 51.8 & 70.2 & 81.7 & 89.6 & 73.33 & 55.7 & 70.9 & 82.5 & 90.5 & 74.90 \\
\hline Asc. at 200 ppm & 59.9 & 81.3 & 93.7 & 105.1 & 85.00 & 60.5 & 82.1 & 94.6 & 106.2 & 85.85 \\
\hline (Sal.+Asc.) at $100 \mathrm{ppm}$ & 64.6 & 87.1 & 101.8 & 112.9 & 91.60 & 65.3 & 88.0 & 102.8 & 114.1 & 92.55 \\
\hline (Sal.+Asc.) at 200 ppm & 50.8 & 68.3 & 79.7 & 87.1 & 71.48 & 51.3 & 68.9 & 81.0 & 88.0 & 72.30 \\
\hline Mean (A) & 54.06 & 60.41 & 84.80 & 93.61 & & 55.10 & 73.49 & 85.71 & 94.59 & \\
\hline \multirow[t]{2}{*}{ L.S.D. at $5 \%$} & \multicolumn{2}{|c|}{ A: 4.83} & B: 4.00 & \multicolumn{2}{|c|}{ AB: 8.00} & \multicolumn{2}{|c|}{ A: 5.66} & B: 4.11 & \multicolumn{2}{|c|}{ AB: 8.22} \\
\hline & \multicolumn{10}{|c|}{ Number of leaves/plant } \\
\hline Control & 8.10 & 9.21 & 9.24 & 9.54 & 9.02 & 9.00 & 10.12 & 10.26 & 10.48 & 9.97 \\
\hline Sal. at 100 ppm & 8.60 & 9.73 & 9.85 & 10.06 & 9.56 & 9.56 & 10.69 & 10.94 & 11.05 & 10.56 \\
\hline Sal. at 200 ppm & 8.79 & 9.94 & 10.07 & 10.29 & 9.77 & 9.77 & 10.92 & 11.18 & 11.31 & 10.80 \\
\hline Asc. at 100 ppm & 8.69 & 9.82 & 9.96 & 10.17 & 9.66 & 9.66 & 10.79 & 11.05 & 11.17 & 10.67 \\
\hline Asc. at 200 ppm & 8.81 & 9.97 & 10.19 & 10.34 & 9.83 & 9.79 & 10.95 & 11.32 & 11.36 & 10.86 \\
\hline (Sal.+Asc.) at $100 \mathrm{ppm}$ & 8.96 & 10.15 & 10.41 & 10.63 & 10.04 & 9.95 & 11.16 & 11.55 & 11.67 & 11.08 \\
\hline (Sal.+Asc.) at 200 ppm & 8.70 & 9.72 & 9.88 & 10.17 & 9.62 & 9.67 & 10.68 & 10.97 & 11.18 & 10.63 \\
\hline Mean (A) & 8.66 & 9.79 & 9.94 & 10.17 & & 9.63 & 10.76 & 11.04 & 11.17 & \\
\hline L.S.D. at 5\% & \multicolumn{2}{|c|}{ A: 0.14} & B: 0.16 & \multicolumn{2}{|c|}{$\mathrm{AB}: 0.32$} & \multicolumn{2}{|c|}{ A: 0.15} & B: 0.16 & \multicolumn{2}{|c|}{$\mathrm{AB}: 0.32$} \\
\hline
\end{tabular}

Sal.: Salicylic acid Asc.: Ascorbic acid 
Table 2. Effect of compost, salicylic and ascorbic acid treatments on leaves dry weight (g) of Gladiolus grandiflorus cv. White Prosperity plants during 2012/2013 and 2013/2014 seasons.

\begin{tabular}{|c|c|c|c|c|c|c|c|c|c|c|}
\hline \multirow{3}{*}{$\begin{array}{l}\text { Salicylic and } \\
\text { ascorbic acids } \\
\text { treatments (B) }\end{array}$} & \multicolumn{10}{|c|}{ Compost levels (ton/feddan) (A) } \\
\hline & \multicolumn{5}{|c|}{$1^{\text {st }}$ Season } & \multicolumn{5}{|c|}{$2^{\text {nd }}$ Season } \\
\hline & $\mathbf{0}$ & 7.5 & 10.0 & 12.5 & Mean (B) & $\mathbf{0}$ & 7.5 & 10.0 & 12.5 & Mean (B) \\
\hline$\overline{\text { Control }}$ & 3.23 & 3.67 & 3.71 & 3.82 & 3.61 & 3.71 & 4.17 & 4.23 & 4.31 & 4.11 \\
\hline Sal. at $100 \mathrm{ppm}$ & 3.44 & 3.88 & 3.94 & 4.03 & 3.82 & 3.94 & 4.41 & 4.51 & 4.64 & 4.38 \\
\hline Sal. at 200 ppm & 3.52 & 3.98 & 4.03 & 4.13 & 3.92 & 4.02 & 4.51 & 4.60 & 4.66 & 4.45 \\
\hline Asc. at $100 \mathrm{ppm}$ & 3.48 & 3.92 & 3.98 & 4.07 & 3.86 & 3.98 & 4.45 & 4.54 & 4.66 & 4.43 \\
\hline Asc. at 200 ppm & 3.62 & 4.08 & 4.17 & 4.23 & 4.03 & 4.05 & 4.52 & 4.67 & 4.79 & 4.51 \\
\hline (Sal.+Asc.) at $100 \mathrm{ppm}$ & 3.69 & 4.18 & 4.29 & 4.38 & 4.14 & 4.10 & 4.62 & 4.76 & 4.81 & 4.57 \\
\hline (Sal.+Asc.) at $200 \mathrm{ppm}$ & 3.48 & 3.88 & 3.95 & 4.07 & 3.85 & 3.97 & 4.39 & 4.53 & 4.61 & 4.38 \\
\hline Mean (A) & 3.49 & 3.94 & 4.01 & 4.10 & & 3.97 & 4.44 & 4.55 & 4.65 & \\
\hline L.S.D. at 5\% & \multicolumn{2}{|c|}{ A: 0.06} & B: 0.08 & \multicolumn{2}{|c|}{ AB: 0.16} & \multicolumn{2}{|c|}{ A: 0.09} & B: 0.06 & \multicolumn{2}{|c|}{ AB: 0.12} \\
\hline
\end{tabular}

Sal.: Salicylic acid $\quad$ Asc.: Ascorbic acid

Hassanein and El-Sayed (2009) and Abdou et al. (2013) on gladiolus.

A possible explanation to the positive effect of compost fertilizer treatments might be attributed to its stimulative effects on different vegetative growth (Tables 1 and 2). Better vegetative growth should be directly reflected on various flowering aspects.

Regarding the effect of salicylic acid and/or ascorbic acid treatments, data in Tables (3 and 4) revealed that all six treatments significantly increased length of spike, rachis length, spike diameter, number of florets/spike, lower florets diameter and single floret fresh weight compared with untreated plants. The highest values for all flowering characters were obtained due to gladiolus plants sprayed with salicylic acid plus ascorbic one, each at $100 \mathrm{ppm}$ followed by ascorbic acid at $200 \mathrm{ppm}$ in both seasons.

This finding was similar to those obtained by Abdel Aziz et al. (2009) and Abo Leila and Eid (2011) on gladiolus and Kasim and Adil (2014) on Freesia hybrida regarding the influence of ascorbic acid treatments. Also, Sajjad et al. (2014) on gladiolus, Khandaker et al. (2011) on red amaranths and Hajizadeh and Aliloo (2013) on Lilium longiflorum regarding the effects of salicylic acid in this concern.
The interaction treatments was exhibited a significant effect for all flowering parameter, except for lower floret fresh weight in both seasons. The highest values were obtained with compost at 12.5 ton/fed in combination with salicylic acid (100 ppm) + ascorbic acid (100 ppm) or ascorbic acid (200 ppm).

\section{REFERENCES}

Abdel Aziz, G.N.; Taha, L. and Ibrahim, S.M.M. (2009). Some studies on the effect of putrescine, ascorbic acid and thiamine on growth, flowering and some chemical constituents of gladiolus at Nubaria. Ozean J. of Applied Sci., 2(2): 169-179.

Abdou, M.A.H.; Aly, M.K. and Ahmed, A.S.A. (2013). Effect of compost, biofertilization and some vitamins addition on Gladiolus grandiflorus. J. Plant Production, Mansoura Univ., 4(12): 1751-1761.

Abo Leila, B. and Eid, R. (2011). Improving gladiolus growth, flower keeping quality by using some vitamins application. Journal of American Science, 7(3):169174.

Anwar, M.; Sahito, H.A.; Hassan, I.; Abbasi, N.A.; Ahmed, H.; Bhatti, M.A.; Hussain, A.; Iqbal, Z. and Abro, A.H. (2014). Effect 
Table 3. Effect of compost, salicylic and ascorbic acid treatments on spike length (cm), rachis length and spike diameter $(\mathrm{mm})$ of Gladiolus grandiflorus cv. White Prosperity plants during 2012/2013 and 2013/2014 seasons.

\begin{tabular}{|c|c|c|c|c|c|c|c|c|c|c|}
\hline \multirow{3}{*}{$\begin{array}{c}\text { Salicylic and } \\
\text { ascorbic acids } \\
\text { treatments (B) }\end{array}$} & \multicolumn{10}{|c|}{ Compost levels (ton/feddan) (A) } \\
\hline & \multicolumn{5}{|c|}{$1^{\text {st }}$ Season } & \multicolumn{5}{|c|}{$2^{\text {nd }}$ Season } \\
\hline & $\mathbf{0}$ & 7.5 & 10.0 & 12.5 & Mean (B) & $\mathbf{0}$ & 7.5 & 10.0 & 12.5 & Mean (B) \\
\hline & \multicolumn{10}{|c|}{ Spike length (cm) } \\
\hline Control & 50.7 & 58.5 & 62.7 & 65.8 & 59.4 & 51.7 & 59.7 & 64.0 & 67.1 & 60.6 \\
\hline Sal. at $100 \mathrm{ppm}$ & 62.6 & 63.9 & 64.7 & 65.9 & 64.3 & 63.8 & 65.6 & 66.7 & 68.0 & 66.0 \\
\hline Sal. at 200 ppm & 65.7 & 67.1 & 67.9 & 69.1 & 67.5 & 68.1 & 71.0 & 72.5 & 73.8 & 71.4 \\
\hline Asc. at 100 ppm & 64.8 & 65.8 & 66.9 & 68.1 & 66.4 & 66.3 & 68.0 & 69.3 & 70.4 & 68.5 \\
\hline Asc. at 200 ppm & 66.6 & 68.2 & 68.9 & 72.2 & 69.0 & 69.9 & 73.1 & 75.7 & 79.4 & 74.5 \\
\hline (Sal.+Asc.) at $100 \mathrm{ppm}$ & 68.9 & 70.6 & 71.4 & 74.8 & 71.4 & 72.4 & 75.6 & 78.3 & 82.1 & 77.1 \\
\hline (Sal.+Asc.) at $200 \mathrm{ppm}$ & 65.2 & 66.4 & 67.3 & 68.5 & 66.9 & 66.9 & 68.7 & 70.1 & 71.3 & 69.3 \\
\hline Mean (A) & 63.5 & 65.8 & 67.1 & 69.2 & & 65.6 & 68.8 & 70.9 & 73.2 & \\
\hline \multirow[t]{2}{*}{ L.S.D. at 5\% } & \multicolumn{2}{|c|}{ A: 1.3} & B: 1.4 & \multicolumn{2}{|c|}{ AB: 2.8} & \multicolumn{2}{|c|}{ A: 1.9} & B: 1.8 & \multicolumn{2}{|c|}{ AB: 3.6} \\
\hline & \multicolumn{10}{|c|}{ Rachis length $(\mathrm{cm})$} \\
\hline Control & 30.4 & 35.1 & 37.6 & 39.5 & 35.7 & 31.0 & 35.8 & 38.4 & 40.3 & 36.4 \\
\hline Sal. at 100 ppm & 38.2 & 39.0 & 39.5 & 40.2 & 39.2 & 38.2 & 39.3 & 40.0 & 40.8 & 39.6 \\
\hline Sal. at 200 ppm & 40.2 & 41.0 & 41.5 & 42.2 & 41.2 & 40.9 & 42.6 & 43.5 & 44.4 & 42.9 \\
\hline Asc. at $100 \mathrm{ppm}$ & 39.5 & 40.1 & 40.8 & 41.5 & 40.5 & 39.7 & 40.8 & 41.5 & 42.0 & 41.0 \\
\hline Asc. at 200 ppm & 40.8 & 41.7 & 42.2 & 44.3 & 42.3 & 42.1 & 44.0 & 45.6 & 47.8 & 44.9 \\
\hline (Sal.+Asc.) at $100 \mathrm{ppm}$ & 42.2 & 43.3 & 43.8 & 45.8 & 43.8 & 43.7 & 45.6 & 47.2 & 49.5 & 46.5 \\
\hline (Sal.+Asc.) at $200 \mathrm{ppm}$ & 39.8 & 40.5 & 41.1 & 41.8 & 40.8 & 40.1 & 41.2 & 42.0 & 42.7 & 41.5 \\
\hline Mean (A) & 38.7 & 40.1 & 40.9 & 42.2 & & 39.4 & 41.3 & 42.6 & 43.9 & \\
\hline \multirow[t]{2}{*}{ L.S.D. at 5\% } & \multicolumn{2}{|c|}{ A: 0.7} & B: 0.9 & \multicolumn{2}{|c|}{ AB: 1.8} & \multicolumn{2}{|c|}{ A: 1.1} & B: 1.0 & \multicolumn{2}{|c|}{ AB: 2.0} \\
\hline & \multicolumn{10}{|c|}{ Spike diameter $(\mathrm{mm})$} \\
\hline Control & 7.4 & 9.4 & 10.1 & 10.3 & 9.3 & 8.1 & 9.1 & 9.6 & 10.0 & 9.2 \\
\hline Sal. at 100 ppm & 9.5 & 11.6 & 12.4 & 12.7 & 11.6 & 11.3 & 12.4 & 12.9 & 13.3 & 12.5 \\
\hline Sal. at 200 ppm & 10.3 & 12.6 & 13.8 & 14.5 & 12.8 & 12.1 & 13.5 & 14.1 & 14.7 & 13.6 \\
\hline Asc. at 100 ppm & 10.0 & 12.2 & 13.1 & 13.5 & 12.2 & 11.5 & 12.7 & 13.1 & 13.6 & 12.7 \\
\hline Asc. at 200 ppm & 10.6 & 13.0 & 14.2 & 15.0 & 13.2 & 12.2 & 13.6 & 14.4 & 15.3 & 13.9 \\
\hline (Sal.+Asc.) at $100 \mathrm{ppm}$ & 10.8 & 13.3 & 14.6 & 15.5 & 13.6 & 12.4 & 13.9 & 14.8 & 15.7 & 14.2 \\
\hline (Sal.+Asc.) at $200 \mathrm{ppm}$ & 10.1 & 12.3 & 13.4 & 14.0 & 12.5 & 11.8 & 13.1 & 13.6 & 14.2 & 13.2 \\
\hline Mean (A) & 9.8 & 12.1 & 13.1 & 13.6 & & 11.3 & 12.6 & 13.2 & 13.8 & \\
\hline L.S.D. at $5 \%$ & \multicolumn{2}{|c|}{ A: 0.5} & B: 0.4 & \multicolumn{2}{|c|}{ AB: 0.8} & \multicolumn{2}{|c|}{ A: 0.06} & B: 0.4 & \multicolumn{2}{|c|}{ AB: 0.8} \\
\hline
\end{tabular}

Sal.: Salicylic acid Asc.: Ascorbic acid 
Table 4. Effect of compost, salicylic and ascorbic acid treatments on number of florets and lower floret diameter and fresh weight of Gladiolus grandiflorus cv. White Prosperity plants during 2012/2013 and 2013/2014 seasons.

\begin{tabular}{|c|c|c|c|c|c|c|c|c|c|c|}
\hline \multirow{3}{*}{$\begin{array}{c}\text { Salicylic and } \\
\text { ascorbic acids } \\
\text { treatments (B) }\end{array}$} & \multicolumn{10}{|c|}{ Compost levels (ton/feddan) (A) } \\
\hline & \multicolumn{5}{|c|}{$1^{\text {st }}$ Season } & \multicolumn{5}{|c|}{$2^{\text {nd }}$ Season } \\
\hline & $\mathbf{0}$ & 7.5 & 10.0 & 12.5 & $\begin{array}{c}\text { Mean } \\
\text { (B) }\end{array}$ & $\mathbf{0}$ & 7.5 & 10.0 & 12.5 & $\begin{array}{c}\text { Mean } \\
\text { (B) }\end{array}$ \\
\hline & \multicolumn{10}{|c|}{ Number of florets/spike } \\
\hline Control & 9.04 & 9.38 & 10.34 & 10.69 & 9.86 & 9.15 & 9.53 & 10.49 & 10.80 & 9.99 \\
\hline Sal. at 100 ppm & 9.43 & 9.84 & 10.86 & 11.23 & 10.34 & 9.62 & 10.01 & 11.02 & 11.34 & 10.50 \\
\hline Sal. at 200 ppm & 9.81 & 10.24 & 11.39 & 11.74 & 10.80 & 10.03 & 10.56 & 11.49 & 11.92 & 11.00 \\
\hline Asc. at 100 ppm & 9.59 & 9.98 & 11.01 & 11.41 & 10.50 & 9.73 & 10.22 & 11.15 & 11.55 & 10.66 \\
\hline Asc. at 200 ppm & 9.99 & 10.43 & 11.58 & 11.95 & 10.99 & 10.24 & 10.77 & 11.69 & 12.11 & 11.20 \\
\hline (Sal.+Asc.) at 100 ppm & 10.29 & 10.74 & 11.89 & 12.27 & 11.30 & 10.57 & 11.09 & 11.99 & 12.48 & 11.53 \\
\hline (Sal.+Asc.) at 200 ppm & 9.63 & 10.03 & 11.18 & 11.53 & 10.59 & 9.85 & 10.37 & 11.28 & 11.70 & 10.71 \\
\hline Mean (A) & 9.68 & 10.09 & 11.18 & 11.55 & & 9.88 & 10.36 & 11.30 & 11.70 & \\
\hline \multirow[t]{2}{*}{ L.S.D. at 5\% } & \multicolumn{2}{|c|}{ A: 0.30} & B: 0.18 & \multicolumn{2}{|c|}{ AB: 0.36} & \multicolumn{2}{|c|}{ A: 0.35} & B: 0.24 & \multicolumn{2}{|c|}{ AB: 0.48} \\
\hline & \multicolumn{10}{|c|}{ lower floret diameter $(\mathrm{cm})$} \\
\hline Control & 7.21 & 7.79 & 8.74 & 8.84 & 8.15 & 7.22 & 7.83 & 8.77 & 8.89 & 8.18 \\
\hline Sal. at 100 ppm & 7.72 & 8.29 & 9.26 & 9.36 & 8.66 & 7.81 & 8.43 & 9.38 & 9.48 & 8.78 \\
\hline Sal. at 200 ppm & 8.13 & 8.68 & 9.65 & 9.74 & 9.05 & 8.20 & 8.87 & 9.80 & 9.94 & 9.20 \\
\hline Asc. at 100 ppm & 7.93 & 8.48 & 9.45 & 9.57 & 8.86 & 8.00 & 8.65 & 9.59 & 9.69 & 8.98 \\
\hline Asc. at 200 ppm & 8.16 & 8.72 & 9.79 & 9.89 & 9.14 & 8.22 & 8.98 & 9.90 & 10.01 & 9.28 \\
\hline (Sal.+Asc.) at $100 \mathrm{ppm}$ & 8.19 & 8.83 & 9.88 & 10.01 & 9.23 & 8.24 & 9.01 & 9.95 & 10.16 & 9.34 \\
\hline (Sal.+Asc.) at 200 ppm & 8.04 & 8.59 & 9.57 & 9.68 & 8.97 & 8.11 & 8.77 & 9.70 & 9.82 & 9.10 \\
\hline Mean (A) & 7.91 & 8.48 & 9.48 & 9.58 & & 7.97 & 8.65 & 9.58 & 9.71 & \\
\hline \multirow[t]{2}{*}{ L.S.D. at 5\% } & \multicolumn{2}{|c|}{ A: 0.11} & B: 0.06 & \multicolumn{2}{|c|}{ AB: 0.12} & \multicolumn{2}{|c|}{ A: 0.14} & B: 0.09 & \multicolumn{2}{|c|}{ AB: 0.18} \\
\hline & \multicolumn{10}{|c|}{ Lower floret fresh weight (g) } \\
\hline Control & 3.65 & 4.75 & 4.89 & 5.30 & 4.63 & 3.81 & 4.91 & 4.93 & 5.37 & 4.76 \\
\hline Sal. at 100 ppm & 4.78 & 5.73 & 5.79 & 6.38 & 5.67 & 4.70 & 5.89 & 5.94 & 6.41 & 5.74 \\
\hline Sal. at 200 ppm & 5.15 & 6.09 & 6.11 & 6.78 & 6.03 & 5.16 & 6.12 & 6.15 & 6.82 & 6.06 \\
\hline Asc. at 100 ppm & 4.99 & 5.91 & 5.98 & 6.59 & 5.87 & 4.95 & 6.01 & 6.09 & 6.69 & 5.94 \\
\hline Asc. at 200 ppm & 5.20 & 6.15 & 6.18 & 6.88 & 6.10 & 5.20 & 6.16 & 6.21 & 6.89 & 6.12 \\
\hline (Sal.+Asc.) at $100 \mathrm{ppm}$ & 5.28 & 6.22 & 6.25 & 6.96 & 6.18 & 5.27 & 6.24 & 6.30 & 6.99 & 6.20 \\
\hline (Sal.+Asc.) at 200 ppm & 5.10 & 6.00 & 6.05 & 6.71 & 5.97 & 5.01 & 6.09 & 6.11 & 6.78 & 6.00 \\
\hline Mean (A) & 4.88 & 5.84 & 5.89 & 6.51 & & 4.87 & 5.92 & 5.96 & 6.56 & \\
\hline L.S.D. at $5 \%$ & \multicolumn{2}{|c|}{ A: 0.51} & B: 0.20 & \multicolumn{2}{|c|}{ AB: N.S. } & \multicolumn{2}{|c|}{ A: 0.38} & B: 0.18 & \multicolumn{2}{|c|}{ AB: N.S. } \\
\hline
\end{tabular}


of pre-harvest treatment of salicylic on growth and vase life of tuberose with aroma environment. J. of Agric. Res., $3(2): 50-57$.

Arfan, M.; Athar, H.R. and Ashraf, M. (2007). Does exogenous application of salicylic acid through the rooting medium modulate growth and photosynthetic capacity in two differently adapted spring wheat cultivars under salt stress. J. Plant Physiol., 6:685-694.

Atta-Alla, H.K.; Zaghloul, M.A. and Hashish, K.H. (2003). Effect of organic manure and NPK fertilizers on vegetative growth, flowering and chemical composition of some Gladiolus cultivars. Ann. Agric. Sci., Moshtohor, 41(2):680691.

Blokhina, O.; Virdainen, E. and Fagersted, K.V. (2003). Antioxidant, oxidative damage and oxygen deprivations stress. A Review Ann. Boi., 91:179-194.

Chandar, I.; Rawat, I.; Lakhawat, S.S. and Yadav, K.K. (2012). Effect of organic manures and biofertilizers on the yield parameters of Gladiolus cv. White Prosperity. Ecol., Environ. and Conservation Paper, 18(1):91-94.

Conte, C.A.M.; Ruppenthal, V.; Zigiotto, D.C.; Bianchini, M.I.F. and Baches, C. (2001). Organic fertilization for the culture of the gladiolus. Scientia Agraria Paranaensis, 1(1):33-41 (C.F. CAB Abst., ENAL).

Dongardive, S.B.; Golliwar, V.J. and Bhongle, S.A. (2007). Effect of organic manure and biofertilizers on growth and flowering in Gladiolus cv. White Prosperity. Plant Archives, 7(2):657-658.

Hajizadeh, H.S. and Aliloo, A.A. (2013). The effectiveness of pre-harvest salicylic acid application on physiological traits in Lilium longiflorum. Inter. J. of Sci. (IJSRES), 1(12):344-350.

Hassanein, Magda M. and El-Sayed, Soheir G. (2009). Effect of some organic and bio-fertilization treatments on gladiolus plants: 1- Vegetative growth and flowering. J. Agric. Sci., Mansoura Univ., 34(6):6237-6254.

Hogan, L. (1990). Bulbs for all seasons. Sunset Western Garden Book, Menlo Park, CA: Lane Publishing, California, pp: 185-198.

Kasim, J.Y. and Adil, A.M. (2014). Effect of gibberellic acid, spraying micronutrient and ascorbic acid on the vegetative growth, flowering of Freesia hybrida cv. Prominence. Journal of Kirkuk University for Agricultural Sciences, 5(1):50-64.

Khan, S.; Khan, A.; Iqbal, A. and Rehman, M. (2002). Effect of different media on growth and quality of gladiolus (Gladiolus hortulanus cv. Jacksonville Gold). Asian J. of plant Sci., 1(6):670671.

Khandaker, L.; Masum Akond, A.S.M.G. and Oba, S. (2011). Foliar application of salicylic acid improved the growth, yield and leaf's bioactive compounds in red amaranth (Amaranthus tricolor, L.). Vegetable Crops Research Bulletin, 74:77-86.

Leonardo, M. and Barbara, D.L. (2011). Organic and mineral soil fertilization in gladiolus. Compost Sci. and utilization, 19(3):178-179.

Maynard, A.A. (1991). Intensive vegetable production using composted animal manures. The Connecticut Agricultural Experiment Station. New Haven, Bulletin, 894.

MSTAT-C (1986). A Microcomputer Program for the Design Management and Analysis of Agronomic Research Experiments (Version 4.0), Michigan State Univ., U.S.A.

Pimpini, F. and Zanin, G. (2002). Gladiolus, Effect of soil type and fertilizer. Culture. Protette, 31(9):107-114. (C.F. www. beds.ac.uk). 
Sajjad, Y.; Jaskani, M.; Ashraf, M.; Qasim, M. and Ahmad, R. (2014). Response of morphological and physiological growth attributes to foliar application of plant growth regulators in Gladiolus "White Prosperity". Pak. J. Agric. Sci., 51(1):123-129.
Zaghloul, M.A.R. and Moghazy, E.I. (2001). Response of some gladiolus cultivars to organic manure and NPK fertilizers in sandy soil under Sinai conditions. Ann. Agric. Sci., Moshtohor, 39(1):585-602.

\section{تأثير معاملات الكمبوست وحامضي الساليسيلك و الأسكوربيك على نمو وإزهار نباتات الجلاديولس جراند فلورس صنف وايت بروسيكيرتي الاسيكي}

محمود عبد الهادي حسن عبده، أحمد عبد المنعم السيد، فتحي عبد اللطيف عطية، أميرة رجائي محمد خليل قسم البساتين، كلية الزر اعة، جامعة المنيا، مصر.

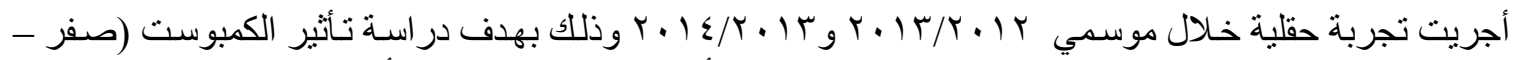

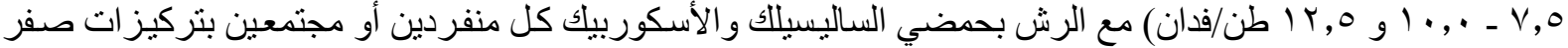

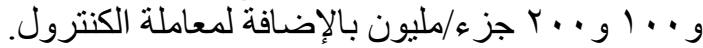

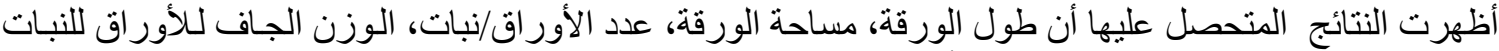

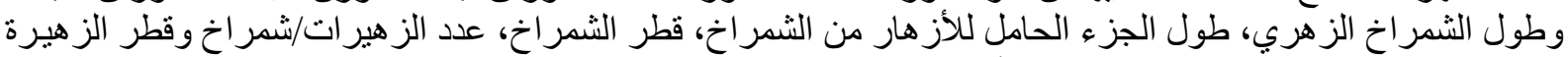

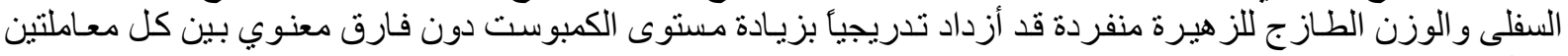

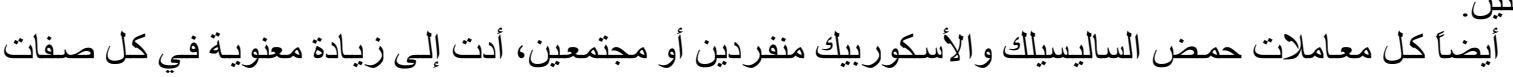

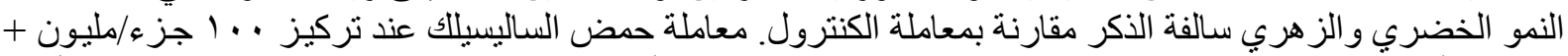

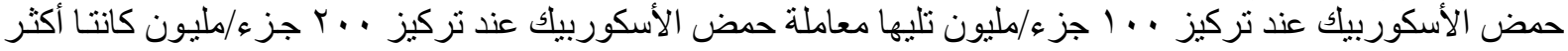

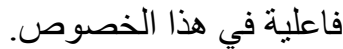

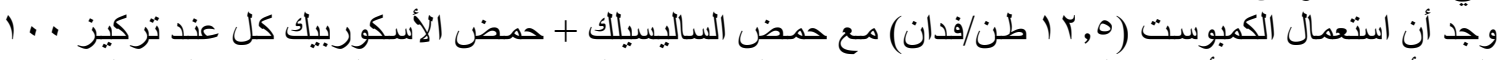

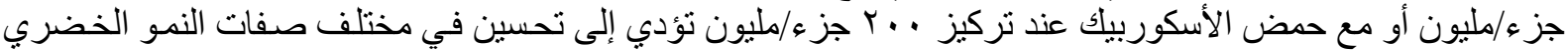

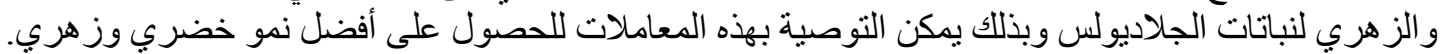


